\title{
Research on a Rotating Machinery Fault Prognosis Method Using Three-Dimensional Spatial Representations
}

\author{
Xiaoni Dong, ${ }^{1,2}$ Xiaodong Zhang, ${ }^{1}$ Zaichao Ma, ${ }^{1}$ Guangrui Wen, ${ }^{1}$ and Zhifen Zhang' \\ ${ }^{1}$ School of Mechanical Engineering, Key Laboratory of Education Ministry for Modern Design and Rotor-Bearing System, \\ Xian Jiaotong University, Xian, China \\ ${ }^{2}$ Xian Siyuan University, Xian, China \\ Correspondence should be addressed to Guangrui Wen; rainy.wen@163.com
}

Received 20 June 2016; Revised 11 September 2016; Accepted 18 October 2016

Academic Editor: Matteo Aureli

Copyright (C) 2016 Xiaoni Dong et al. This is an open access article distributed under the Creative Commons Attribution License, which permits unrestricted use, distribution, and reproduction in any medium, provided the original work is properly cited.

\begin{abstract}
Process models and parameters are two critical steps for fault prognosis in the operation of rotating machinery. Due to the requirement for a short and rapid response, it is important to study robust sensor data representation schemes. However, the conventional holospectrum defined by one-dimensional or two-dimensional methods does not sufficiently present this information in both the frequency and time domains. To supply a complete holospectrum model, a new three-dimensional spatial representation method is proposed. This method integrates improved three-dimensional (3D) holospectra and 3D filtered orbits, leading to the integration of radial and axial vibration features in one bearing section. The results from simulation and experimental analysis on a complex compressor show that the proposed method can present the real operational status and clearly reveal early faults, thus demonstrating great potential for condition-based maintenance prediction in industrial machinery.
\end{abstract}

\section{Introduction}

A key objective of condition-based maintenance (CBM) is to determine the optimal time for the replacement or overhaul of a machine. The prediction ability of a machine system is crucial for operation, productivity improvement, service time extension, and system safety enhancement [1]. It is obvious that a complex mechanical system includes a large amount of rotating machinery coupled with intricate structures and multisource excitation. To date, numerous prognostic models have been proposed in the technical literature. Most prognostic methods encounter the problem of long-term prediction for ideal industrial applications. However, we note that the existing techniques encounter difficulty in solving problems in our fields due to the stochastic process that accompanies failure events. Typically attributed to periodical centrifugal force, the compressor rotor usually precesses at a high speed, causing transverse forced vibration that affects smooth running. As a result, it is difficult to observe the running condition using convention signal-processing techniques.
To easily depict the status of the rotating machinery, the holospectrum theory $[2,3]$ and its orbit extension have been proposed, as first introduced by Qu at Xian Jiao Tong University. This approach has been widely used in rotor monitoring and fault diagnosis. Based on the conventional FFT spectra, the holospectral technique accurately identifies the amplitude, phase, and frequency of the vibration signal using interpolation techniques. The vibration condition can be shown by fusing the horizontal and vertical information from one section [3]. Applications to rotating machinery have been published $[4,5]$. To date, the $2 \mathrm{D}$ holospectrum, 3D holospectrum, holowaterfall plot, and short-time complex spectrum have been developed to extend the holospectral techniques [6]. Based on the 2D holospectrum, the 3D holospectrum was established to comprehensively reflect the entire vibrational status of a rotor by observing a series of rotating frequency ellipses. Shi et al. proposed a rotor vibration model decomposition method based on three-dimensional holographic spectral decomposition. This method verified that the rotor vibration mode is the superposition of the firstand second-order mode caused by the force and the couple 
imbalance, respectively [7-11]. Subsequently, new dynamic balancing methods were developed by combining the $3 \mathrm{D}$ holospectrum with intelligent optimization methods. Liu and $\mathrm{Qu}$ [12] proposed a holobalancing method based on the genetic algorithm that transforms the balance equation into an optimization problem. By coding and setting the fitness function, the optimal solution searches in the global space. Wen et al. [13] proposed a holobalancing method based on particle swarm optimization, which searches for the optimal equilibrium position by changing the population location. To correct the error of the holospectrum at the condition of speed fluctuation, order analysis techniques have been introduced into the holospectrum in recent years [14, 15]. The order analysis technique is able to accurately extract the characteristic of the investigated signal with conditions of nonstationarity, thus obtaining the corresponding spectrum information. Wang et al. [16] proposed a FT refinement correction order spectrum method that obtains the accurate amplitude and phase of each order. Wen et al. [17] proposed the holowaterfall plot based on Fode Kaman order tracking, which realizes transient balancing by extracting the fault features in the startup and shutdown stages. Li et al. [18] introduced EMD to assist in holospectrum fault identification. However, the conventional holospectrum can show only a single order of the frequency component $[19,20]$. Additionally, because of the complexity of the rotor vibration characteristics and serious disturbance from noise, errors frequently exist in the orbit diagram. The primary problem is that different types of orbits may display similar features. Thus, it is difficult to deliver effective diagnostic information using the original orbit and its identification method [21, 22].

In this paper, we propose a new three-dimensional spatial representation method. As such, the three-dimensional axis orbit introduces the rotor axial vibration to complement the conventional holospectrum. This element contains additional information on the rotor vibration, thus reflecting the state of the rotor vibration more comprehensively. By displaying the three-dimensional axis orbit, the overall rotor vibration is easily observed, and the failure mechanism of the rotor system can also be carefully analyzed. Therefore, using clearer vibration process information, the fault diagnosis process can be simplified, and, as a result, fault diagnosis of rotating machinery is expected to become more efficient and accurate, thus improving the capability of fault identification and promoting the prediction techniques.

The remainder of this paper is organized as follows. In Section 2, the related concept of the holospectrum and the finite impulse response filter are described in brief. The original contribution of the paper is presented in Section 3, in which we offer a detailed description of rotor vibration analysis in the three-dimensional spatial representation, including the three-dimensional vibration analysis principle and the three-dimensional axis orbit. In Section 4, we briefly illustrate the general information for a rotor test bench and present the misalignment experimental results to validate the feasibility of the proposed method. The method is also verified by engineering application of rotating machinery in Section 5. Conclusions and possible extensions are given in Section 6.

\section{Basic Concept}

2.1. Holospectrum. The holospectrum fuses information from a multisensor to unit fault monitoring and diagnosis. Based on the FFT algorithm, this approach uses interpolation to obtain accurate amplitude, frequency, and phase values of a vibration signal and combines these three types of information in both the horizontal and vertical directions. The holospectrum uses a series of synthetic ellipses to describe the vibrations of the rotor produced by different frequency components and applies the information from both a singlesection of the multibearing rotor system and the overall shaft vibration, which are integrated into comprehensive diagnostic information. Moreover, this method creates fusion of the information in the frequency domain and mainly targets the stationary signal. Figure 1(a) shows the structural diagram of the two-dimensional holospectrum (the axes are marked with rotation, counterclockwise precession of + , and clockwise precession of -). The three-dimensional holospectrum is established by integrating the two-dimensional holospectrum according to the corresponding time at each section. Figure 1(b) shows the structural diagram of the threedimensional holospectrum.

The two-dimensional holospectrum primarily targets the stationary signal of a rotor. Assuming that the parameter coordinates of the $i$ th component of the vibration signal are described by

$$
\begin{aligned}
& x_{i}=A_{i} \sin \left(i \omega t+\alpha_{i}\right), \\
& y_{i}=B_{i} \sin \left(i \omega t+\beta_{i}\right),
\end{aligned}
$$

then

$$
\begin{aligned}
& x_{i}=\left(A_{i} \cos \alpha_{i}\right) \sin (\omega t)+\left(A_{i} \sin \alpha_{i}\right) \cos (\omega t), \\
& y_{i}=\left(B_{i} \cos \beta_{i}\right) \sin (\omega t)+\left(B_{i} \sin \beta_{i}\right) \cos (\omega t) .
\end{aligned}
$$

Or

$$
\begin{aligned}
& x_{i}=s x_{i} \sin (\omega t)+c x_{i} \cos (\omega t), \\
& y_{i}=s y_{i} \sin (\omega t)+c y_{i} \cos (\omega t) .
\end{aligned}
$$

The initial point is as follows:

$$
\begin{aligned}
& x_{o i}=A_{i} \sin \alpha_{i}, \\
& y_{o i}=B_{i} \sin \beta_{i},
\end{aligned}
$$

where $\alpha_{i}$ and $\beta_{i}$ are the initial phases of the $i$ th component.

If $\omega$ is known, the frequency ellipse of any measuring surface can be represented by $r_{i}=\left[s x_{i}, c x_{i}, s y_{i}, c y_{i}\right]$. The threedimensional holospectrum integrates all of the frequency ellipses at the supporting points such that the parameter matrix can be written as

$$
\mathbf{R}=\left[\begin{array}{c}
r_{1} \\
r_{2} \\
\vdots \\
r_{n}
\end{array}\right], \quad i=1,2, \ldots, n,
$$




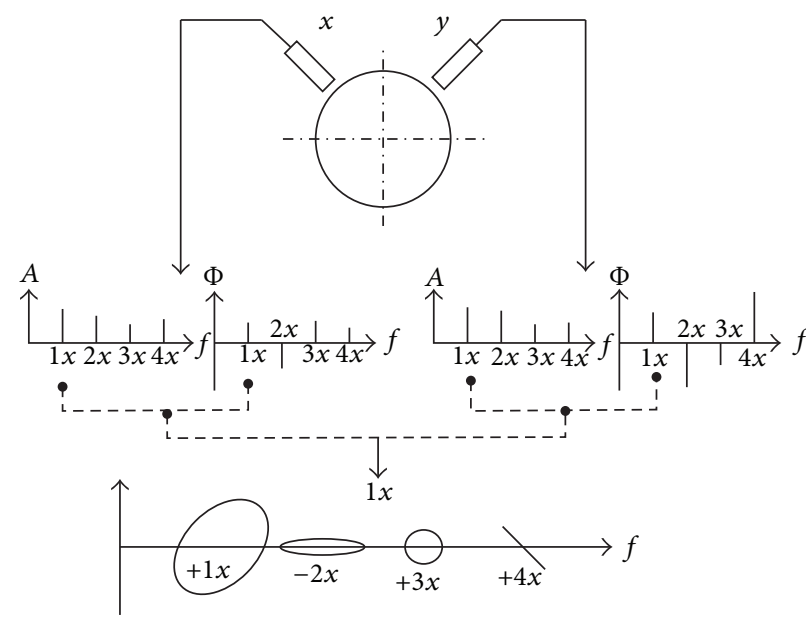

(a) Two-dimensional holospectrum

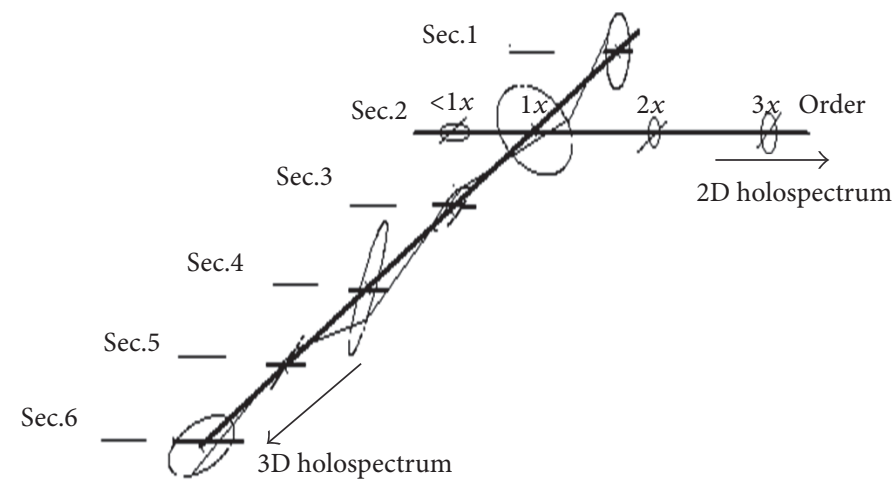

(b) Three-dimensional holospectrum

FIGURE 1: Structure of holospectrum.

where $n$ is the number of the support sections. The initial phase point matrix is

$$
\mathbf{I P P}=\left[\begin{array}{cc}
c x_{1} & c y_{1} \\
c x_{2} & c y_{2} \\
\vdots & \vdots \\
c x_{n} & c y_{n}
\end{array}\right] .
$$

The two-dimensional holospectrum delivers the amplitude of the rotor vibration and also reflects the relationship of the phase with the two directions. With highly integrated amplitude, frequency, and phase, the specific vibration information of the rotor is described as follows:

(1) Shapes of vibration components, such as circle, approximate circle, ellipse, and straight line.

(2) Major axis of the ellipse, the eccentricity, and the angle between the major axis and the coordinate axes.

(3) Clockwise and counterclockwise precession of the rotation direction of the vibration components.

(4) Initial phase of the vibration components.

(5) The relationship between the vibration components, such as the angle between the major axis of each vibration component and the similarity of the shapes.

In this paper, we propose the three-dimensional holospectrum technique, which displays the hybrid vibration modes of the rotor at the working speed. Moreover, this technique also delivers a wealth of information, such as a full view of the rotor vibration, the rigidity of the bearing, the largest component of the vibration, and the force situation of the rotor, which are difficult to obtain by other methods.

2.2. Finite Impulse Response (FIR) Filter. The FIR digital filter plays an important role in digital signal processing and has been widely applied [23]. This filter can be used to achieve band-pass filtering of an investigated signal. Assuming that the investigated signal is $x(t)$, after preprocessing, the signal can be transformed into a discrete form of $x(n)$. If the impulse response of the FIR filter is a finite-length sequence with real number $h(n)$, the output signal can be written as

$$
y[n]=\sum_{k=0}^{N-1} h[k] x[n-k] .
$$

If we perform a $z$ transform on (8), the system transfer function $H(z)$ can be obtained as shown:

$$
H(z)=\sum_{n=0}^{N-1} h[n] z^{-n}
$$

$$
\begin{aligned}
& \text { If } z=e^{j \omega} \text {, then } \\
& \qquad H(\omega)=\sum_{n=0}^{N-1} h[n] e^{-j \omega n} .
\end{aligned}
$$

For the periodicity of $e^{-j \omega n}, H(\omega)$ is the periodic function of the continuous frequency $\omega$ with a $2 \pi$ cycle, and it can be expressed in the polar form:

$$
H(\omega)=|H(\omega)| e^{j \phi(\omega)},
$$

where $H(\omega)$ and $\phi(\omega)$ are the filter frequency and phasefrequency response, respectively.

The design of the FIR filter identifies a discrete-time system with a finite-length unit sample response $h(n)$ according to the desired frequency response $H_{d}(\omega)$. If $h_{d}(n)$ is the unit sample response of the needed filter, then

$$
H_{d}(\omega)=\sum_{n=-\infty}^{\infty} h_{d}[n] e^{-j n \omega} .
$$

The aim is to use $H(\omega)$ in (11) to approximate $H_{d}(\omega)$. The easiest method is the window function approximation 
in which the window function $\omega(n)$ of the time domain multiplies the infinitely long unit sample response $H_{d}(n)$, and the infinitely long unit sample response order is truncated. All of the above constitute the FIR digital filter $h(n)$ :

$$
h[n]=H_{d}[n] \omega(n) .
$$

Finally, the frequency characteristic $H(\omega)$ of the filter can be calculated by $h(n)$.

\section{Design of Rotor Vibration Analysis in a Three-Dimensional Spatial Representation}

3.1. Three-Dimensional Vibration Analysis Principle. The three-dimensional vibration analysis extracts the vibration amplitude and phase information in a three-dimensional holographic spectrum under all of the orders of the frequency component. By synthesizing according to need, we finally construct a synthetic three-dimensional vibration from the selected frequency components. The vector expressions are shown in (7):

$$
\vec{Z}=\sum_{i}^{M} \vec{Z}_{i}
$$

where $M$ is the highest order of frequency.

Three-dimensional vibration analysis includes a filtering process and synthetic three-dimensional vibration analysis. The filtered three-dimensional vibration is used to extract vibration characteristics with a continuous band. The synthetic three-dimensional vibration integrates the required frequency components and reflects the failure features better than single frequency analysis.

3.1.1. Three-Dimensional Vibration Filtering. A continuous colored frequency band is commonly used to depict the fault features in vibration analysis of rotating machinery. The characteristics of this continuous band signal must be studied when failures appear. In our method, filtering is the first step for the investigated signal. The lower and upper limitations are selected by the band-pass filtering method.

In this step, the band-pass filter is designed. From the perspective of the unit sample response, the digital filter can be classified into the infinite impulse response (IIR) and finite impulse response (FIR). Although the IIR filter is better for searching the amplitude-frequency characteristic, the linear phase characteristics may be lost. In the process of signal transformation, distortion of the phase could cause the investigated signal to fail. Due to the strict linear phase characteristic, the FIR filter is commonly used in the field of signal transformation. Furthermore, the impulse response of the FIR filter is a finite-length sequence, the transfer function is a finite polynomial, and the structure is given in a nonrecursive form. As a result, the FIR is always stable. After finite precision computation, the quantitative effect of the parameters is not serious.

According to Section 2.2, the filter effects are obtained using different windows. The window function is expected to enhance the frequency characteristic of the filter and reduce signal leakage error. Thus, the sidelobe should be as small as possible, and the main lobe should also be as narrow as possible. However, we know that these two requirements usually contradict each other. The common method used to suppress the sidelobe is to increase the width of the main lobe. The conventional window functions used in the FIR filter are listed as follows: rectangular window, Haining window, Hamming window, Kaiser window, and so forth. If we use the Hamming window, also known as the improved risingcosine window, the energy ratio is maintained at $99.6 \%$ for the maximal main lobe. Moreover, the width of the sidelobe is as small as the main lobe. Consequently, the Hamming window is used to approximate the filtering function. The expression is shown as follows:

$$
\omega[n]= \begin{cases}\frac{[1-\cos (2 \pi n /(N-1))]}{2} & 0 \leq n \leq N-1 \\ 0 & \text { else. }\end{cases}
$$

The filtered signal contains the signal with the selected frequency band, which presents the filtering three-dimensional vibration analysis.

3.1.2. Synthetic Three-Dimensional Vibration. The synthetic three-dimensional vibration superimposes the threedimensional holospectrum with the specific frequencies. The three-dimensional holospectrum of the $i$ th order is shown as follows:

$$
\vec{Z}_{i}=\vec{Z}_{1 i}+\vec{Z}_{2 i}+\cdots+\vec{Z}_{n i}
$$

where $n$ is the number of rotor sections.

If the $m$ th frequency component must be synthesized, it can be expressed using the array form shown in the following:

$$
\omega[n]=\left\{\begin{array}{l}
\vec{Z}_{1}=\vec{Z}_{11}+\vec{Z}_{21}+\cdots+\vec{Z}_{n 1} \\
\vec{Z}_{2}=\vec{Z}_{12}+\vec{Z}_{22}+\cdots+\vec{Z}_{n 2} \\
\vdots \\
\vec{Z}_{m}=\vec{Z}_{1 m}+\vec{Z}_{2 m}+\cdots+\vec{Z}_{n m} .
\end{array}\right.
$$

The expression of the synthetic three-dimensional vibration is shown as follows:

$$
\vec{Z}_{\text {synthetic }}=\vec{Z}_{1}+\vec{Z}_{2}+\cdots+\vec{Z}_{m} .
$$

A certain amount of fault features requires multiple frequency components to be observed rather than a single one. Taking misalignment as an example, the vibration amplitude and phase from one order are not sufficient to clearly recognize this fault. As a result, the synthetic threedimensional vibration that displays the entire rotor condition is better for fault recognition.

3.2. Analysis of Three-Dimensional Axis Orbit. Due to failure caused by axial friction, it is necessary to add an axial displacement sensor to detect the axial vibration signal. To 


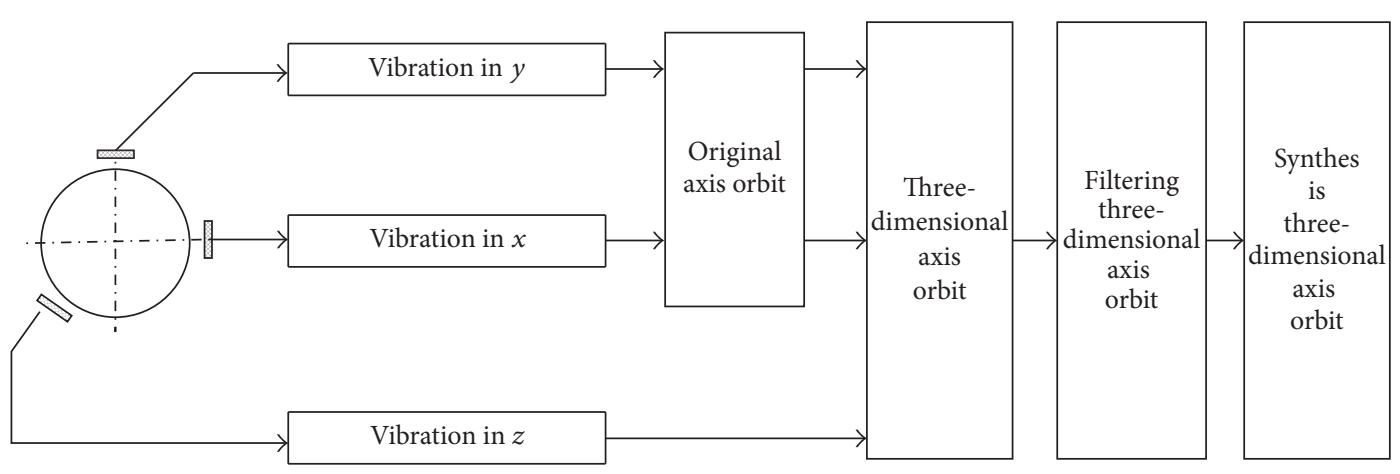

FIgURE 2: Axis trajectory analysis method in a three-dimensional process.
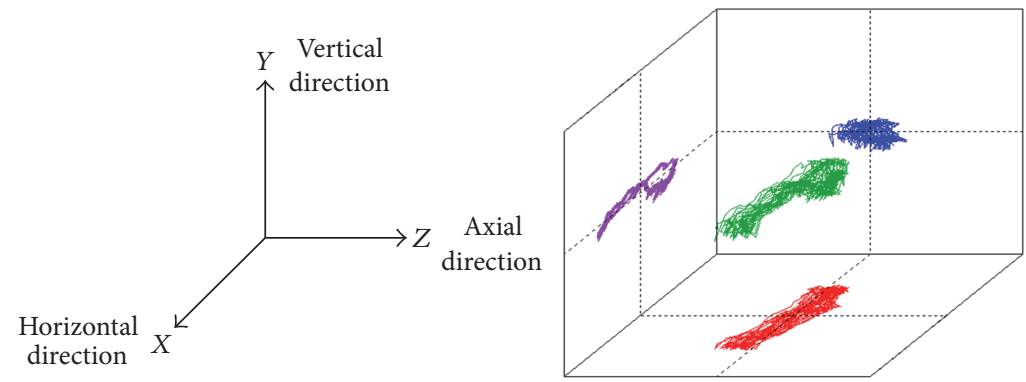

FiguRE 3: Three-dimensional axis orbit diagram.

clearly display the rotor fault according to the vibration signal, the vibration signal that represents the axis orbit must be filtered and purified. With the combination of the bearing three-dimensional axis orbit [24], this paper proposes the three-dimensional filtering axis orbit and three-dimensional synthetic axis orbit. The three-dimensional axis orbit integrates the three directions of movement information with the rotor detection-section central point. The noise interference involved in the signal can be reduced using the threedimensional filtering axis orbit and the three-dimensional synthetic axis orbit. The axis orbit analysis process for the three-dimensional axis orbit is shown in Figure 2.

According to the synthetic methods of the axis trajectory, the instantaneous amplitudes of the signal collected by the three sensors are taken as $(x, y, z)$ in the spatial $X-Y-Z$ directions using one-to-one correspondence. With the continuous running process, this point formats the threedimensional axis orbit in $X-Y-Z$ space. We assume that the investigated signals acquired by the sensors are $x(t), y(t)$, and $z(t)$, and $w(t)$ are the combined three-dimensional axis orbits in $X-Y-Z$ space ( $t$ as parameter). The expression is shown as follows:

$$
w(t)=i x(t)+j y(t)+k z(t) .
$$

The vector expression of (10), as shown in (11), is given as follows:

$$
\vec{W}=\vec{X}+\vec{Y}+\vec{Z}
$$

Equation (19) describes the three-dimensional axis orbit and contains the rotor vibration information in three directions, thus forming a three-dimensional spatial pattern. Different faults in different measuring directions have different reflections. For example, the unbalanced fault vibration is more obvious in the horizontal direction, and the misalignment fault shows significant axial displacement. Therefore, the three-dimensional axis orbit can be projected into the two-dimensional plane, which makes the three-dimensional axis orbit more clearly observable. The diagram of threedimensional axis orbit of section of a rotor is shown in Figure 3.

The overall rotor vibration can be observed using this display of the three-dimensional axis orbit. The result of the proposed method is similar to the single-section in a three-dimensional axis orbit. For example, the adopted vector expression for the axis orbit of two sections with threedimensional characteristics is shown as follows:

$$
\begin{aligned}
& \vec{W}_{1}=\vec{X}_{1}+\vec{Y}_{1}+\vec{Z}_{1}, \\
& \vec{W}_{2}=\vec{X}_{2}+\vec{Y}_{2}+\vec{Z}_{2},
\end{aligned}
$$

where $X_{1}, Y_{1}$, and $Z_{1}$ and $X_{2}, Y_{2}$, and $Z_{2}$ correspond to the signal vector in two cross sections in the horizontal, vertical, and axial directions, respectively. Figure 4 shows a schematic diagram of two sections with a three-dimensional axis orbit. Thus, the failure mechanism of the rotor system can be analyzed by observing the synthesis of multiple sections in the three-dimensional axis orbit. 


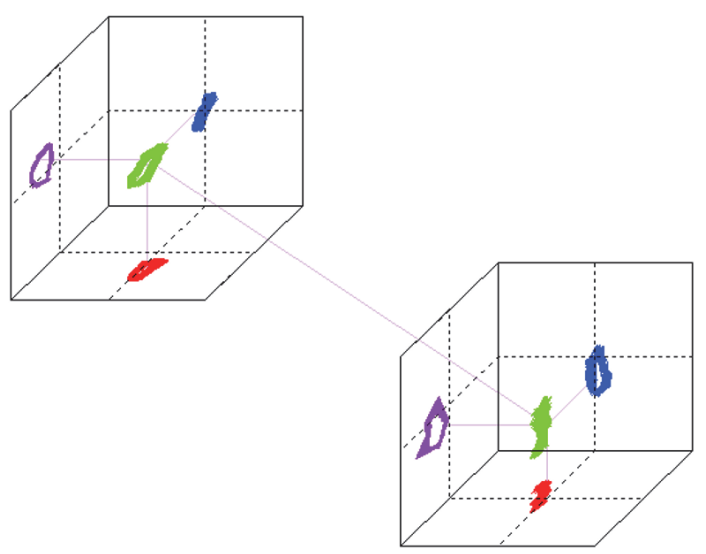

FIGURE 4: Two-section three-dimensional axis orbit.

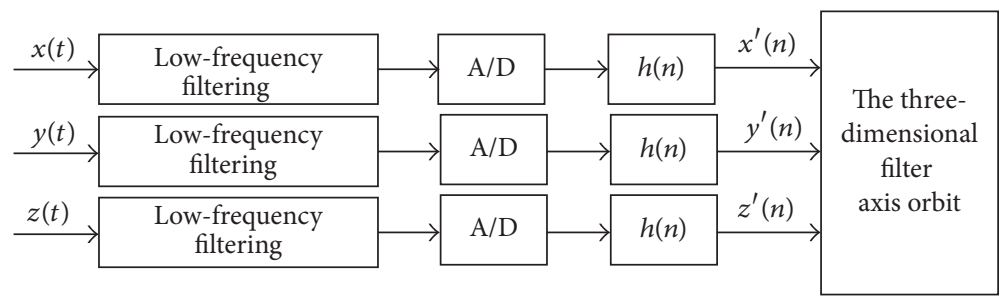

FIGURE 5: Diagram of the three-dimensional filtering orbit.

In summary, the three-dimensional axis orbit introduces rotor axial vibration to complement the conventional holospectrum. Compared with the common twodimensional axis orbit, the three-dimensional axis orbit can reflect the state of rotor vibration more comprehensively.

3.2.1. Three-Dimensional Filtering Orbit. Due to the large transmission distance and dynamic electrical interference, the analog signal is often mixed with a variety of noise interferences that distort the sampled data and seriously impede vibration detection analysis. Therefore, filters must be used to remove the noise interference from the original signal.

In constructing a three-dimensional filtering orbit, the vibration signals $x(t), y(t)$, and $z(t)$ should be filtered first. Subsequently, the three-dimensional orbit can be reconstructed with the filtered signals $x(n), y(n)$, and $z(n)$ in the time domain. The process of implementation of the threedimensional filter orbit is shown in Figure 5.

In this process, $x(n), y(n)$, and $z(n)$ are the discretized sample values of the signals $x(t), y(t)$, and $z(t)$, respectively, created by low-pass filtering. In detail, $h(n)$ is the impulse response of the filter, and $x(n), y(n)$, and $z(n)$ are the filtered signals. The expressions of the filtered signals are shown as follows:

$$
\begin{aligned}
& x(n)=x(t) * h(n), \\
& y(n)=y(t) * h(n), \\
& z(n)=z(t) * h(n) .
\end{aligned}
$$

Using (10) to calculate the filtered signal, we can obtain the three-dimensional filtered orbit. In this work, we show a comparison between the filtered three-dimensional orbit and the original three-dimensional orbit. The schematic diagrams are shown in Figure 6.

In Figure 6(a), the graph lines are complicated and confused with noise, and the characteristic running condition is not obvious. However, in Figure 6(b), the track with noise restores the original appearance, and the filtered orbit with clear graphic lines is superposed with several ellipses. Therefore, the three-dimensional filtered orbit is suitable for analyzing the rotor vibration signal with continuous band features.

3.2.2. Synthetic Three-Dimensional Orbit. Generally, the rotor fault is composed of a dominant vibration and several adjoint components. In addition to the synchronous vibration component caused by unbalance, the vibration signal usually includes subsynchronous vibration components whose frequencies are lower than the rotating frequency and higher harmonic components whose frequencies can be presented as an integer multiple of the rotation speed. These rules easily produce a complex and confused rotor orbit and even cause great difficulties in identifying the orbit. To overcome the difficulty in searching the original orbit, the vibration signal is decomposed into a series of simple harmonic motion components based on the principle of spectral analysis, and the desired frequencies can be integrated. Finally, the synthetic orbit creates clearer feature information for fault identification. 


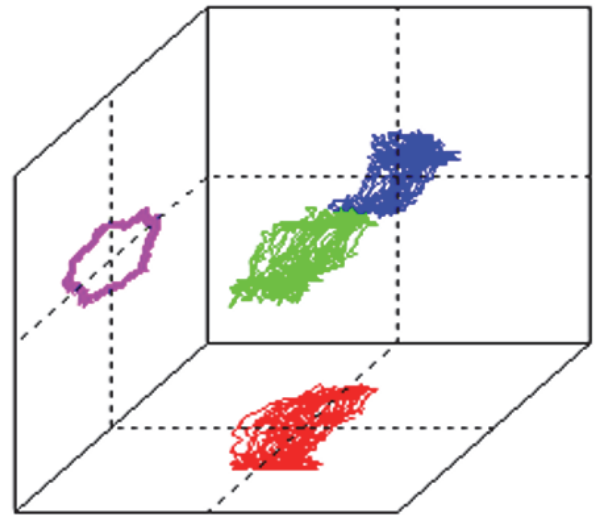

(a) Original three-dimensional orbit

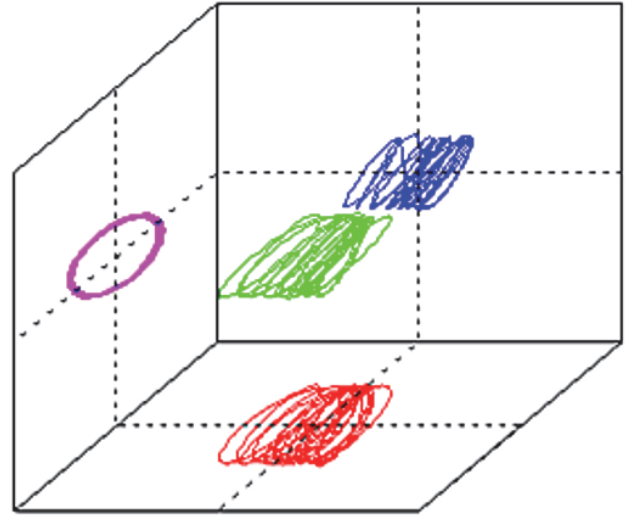

(b) Filtered three-dimensional orbit

FIGURE 6: Original and filtered three-dimensional orbit.

Suppose that $x(t), y(t)$, and $z(t)$ are the rotor vibration signals in the respective $X, Y$, and $Z$ directions, and these three direction signals can be decomposed into several harmonic vibration components, as shown in the following:

$$
\begin{aligned}
x(t)= & A_{1} \sin \left(\omega_{0} t+\phi_{1}\right)+A_{2} \sin \left(2 \omega_{0} t+\phi_{2}\right)+\cdots \\
& +A_{n} \sin \left(n \omega_{0} t+\phi_{n}\right) \\
= & x_{1}(t)+x_{2}(t)+\cdots+x_{n}(t), \\
y(t)= & B_{1} \sin \left(\omega_{0} t+\psi_{1}\right)+B_{2} \sin \left(2 \omega_{0} t+\psi_{2}\right)+\cdots \\
& +B_{n} \sin \left(n \omega_{0} t+\psi_{n}\right) \\
= & y_{1}(t)+y_{2}(t)+\cdots+y_{n}(t), \\
z(t)= & C_{1} \sin \left(\omega_{0} t+\xi_{1}\right)+C_{2} \sin \left(2 \omega_{0} t+\xi_{2}\right)+\cdots \\
& +C_{n} \sin \left(n \omega_{0} t+\xi_{n}\right) \\
= & z_{1}(t)+z_{2}(t)+\cdots+z_{n}(t)
\end{aligned}
$$

where $A_{n}, B_{n}, C_{n}, \phi_{n}, \psi_{n}$, and $\xi_{n}$ are the amplitudes and phases of the signal in the horizontal, vertical, and axial directions, $\omega_{0}$ is the rotational angular frequency of the signal, and $n$ is the highest order of the frequency component.

The components $x_{i}(t), y_{i}(t)$, and $z_{i}(t)$ are composited equations, as shown in the following:

$$
\begin{aligned}
& x_{1}(t)=A_{1} \sin \left(\omega_{0} t+\phi_{1}\right), \\
& y_{1}(t)=B_{1} \sin \left(\omega_{0} t+\psi_{1}\right), \\
& z_{1}(n)=C_{1} \sin \left(\omega_{0} t+\xi_{1}\right), \\
& x_{2}(t)=A_{2} \sin \left(\omega_{0} t+\phi_{2}\right), \\
& y_{2}(t)=B_{2} \sin \left(\omega_{0} t+\psi_{2}\right),
\end{aligned}
$$

$$
\begin{gathered}
z_{2}(n)=C_{2} \sin \left(\omega_{0} t+\xi_{2}\right), \\
\vdots \\
x_{n}(t)=A_{n} \sin \left(\omega_{0} t+\phi_{n}\right), \\
y_{n}(t)=B_{n} \sin \left(\omega_{0} t+\psi_{n}\right), \\
z_{n}(n)=C_{n} \sin \left(\omega_{0} t+\xi_{n}\right) .
\end{gathered}
$$

It can be observed from 3.2.2 that the complex orbit is superposed by different frequencies, phases, and amplitude components. The synthetic orbit expression can be obtained by synthesizing every component, as shown in the following:

$$
\begin{aligned}
c(t)= & i x(t)+j y(t)+k z(t) \\
= & i \cdot \sum_{l}^{n} A_{l} \sin \left(l \omega_{0} t+\phi_{l}\right)+j \\
& \cdot \sum_{l}^{n} B_{l} \sin \left(l \omega_{0} t+\psi_{l}\right)+k \\
& \cdot \sum_{l}^{n} C_{l} \sin \left(l \omega_{0} t+\xi_{l}\right),
\end{aligned}
$$

where $l$ is the frequency component that must be synthesized.

The schematic diagrams shown in Figure 7 illustrate the difference between the original and synthetic threedimensional spatial orbits.

It can be observed from Figure 7 that the synthetic threedimensional orbit shows clear curve features, whereas the original three-dimensional orbit displays fuzzy shapes. In the field of fault diagnosis for rotating machinery, a certain types of faults always produce strong axial vibration, such as misalignment. At this point, if we apply the axial information in a suitable manner, the fault diagnosis process is simplified. Thus, we apply the different shape features described by the 


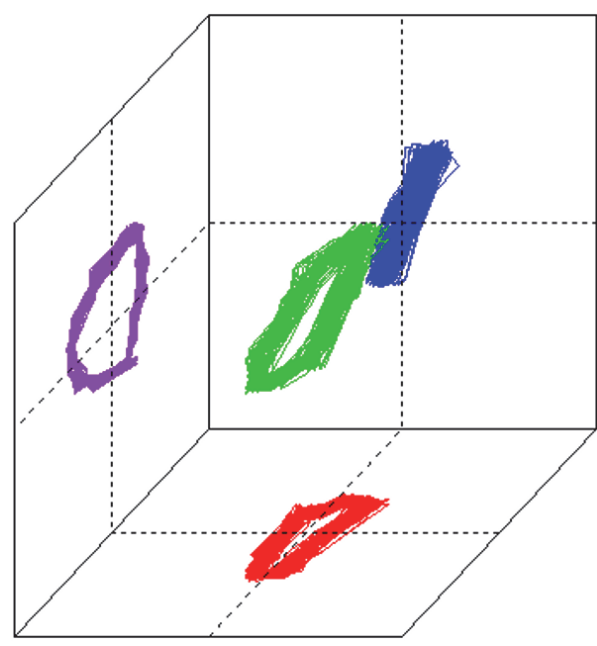

(a) Original three-dimensional orbit

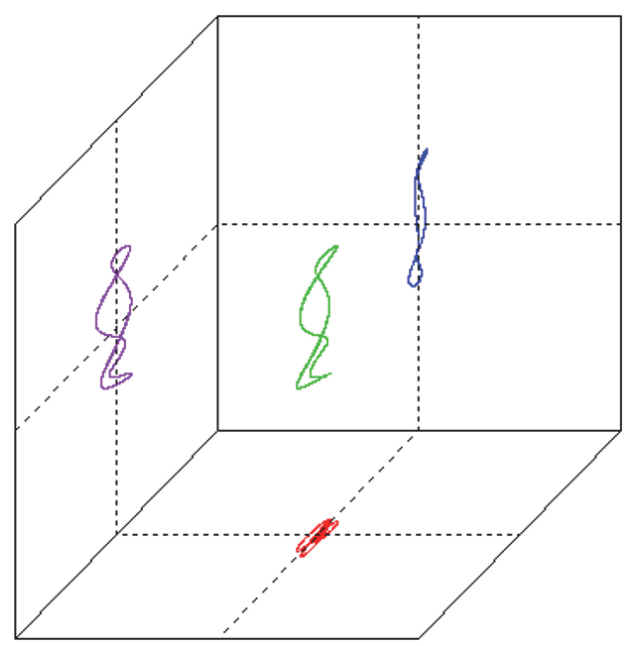

(b) Synthetic three-dimensional orbit

FIGURE 7: Original and synthetic three-dimensional spatial orbit.

synthetic three-dimensional orbit to recognize the failure characteristics.

\section{Experimental Apparatus and Data Analysis}

In this paper, the Bently RK4 rotor test bench is applied to further verify the proposed three-dimensional spatial representation in practical signal analysis. The structure of the experimental platform and the axial misalignment data analysis is presented in the following sections.

4.1. Brief Introduction to the Rotor Test Bench. This platform primarily consists of a rotor system and a vibration testing system. The rotor system includes a rotor, a motor, a pair of bearings, and a foundation. The vibration testing system includes six groups of eddy current sensors and a set of data acquisition instruments connected to a computer. In detail, sensors 1-4 are divided into two groups for capturing the vibration signals located in the cross-section with the directions of $45^{\circ}$ and $135^{\circ}$. Sensor 5 is used to measure phase, and sensor 6 is used to obtain the rotating speed. The main framework of Bently RK4 rotor test bench is shown in Figure 8.

4.2. Fault Identification of Axial Misalignment. The misalignment fault is one of the common rotor faults. This fault is not obvious if we observe the only the vibration characteristics with the order frequency components. The three-dimensional holographic spectra of two cross sections in the $1 X, 2 X$, and $3 X$ components are shown in Figures 9(a)9 (c), respectively. The synthetic three-dimensional vibration is shown in Figure 9(d).

In Figure 9, the first three Figures 9(a)-9(c) show only the ellipse with different sizes and shapes, and Figure 9(d) shows that the two sections appear in the form of a figure " 8 ." In detail, the size of the ellipse with $1 X$ is normal. However, the size of the ellipse with $2 X$ is much larger with low eccentricity.

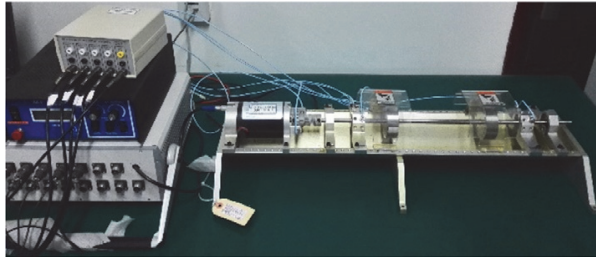

FIGURE 8: Bently RK4 rotor test bench.

Furthermore, the size of the ellipse with $4 X$ is notably small. These three types of information are the primary features of misalignment. As observed, our synthesized graphics can integrate the primary vibration information and fully present the features of the running condition. According to the vibration mechanism, if the synthesis frequency components of $1 X, 2 X$, and $4 X$ appear in the form of a figure " 8 ", the current rotor fault is predominantly misalignment. Therefore, the synthesis of three-dimensional vibration analysis can display the fault features more clearly and conveniently.

\section{Engineering Application}

Fluid-induced vibration is a type of low-frequency vibration excited by fluid acting on rotor. This low-frequency vibration is always expressed as a colored noise band on the spectrum. To address a common fault that appears in turbo-machinery, a certain plant applied our method to analyze fluid-induced vibration. A chart of the filtering three-dimensional vibration on a rotor with two sections collected by the analysis software is shown in Figure 10.

In Figure 10, we observe two groups of ellipses with a stable center of gravity and an unstable amplitude in the filtered three-dimensional vibration projected in a twodimensional plane. These features are typically included in fluid-induced vibration. As a result, the fault caused by 


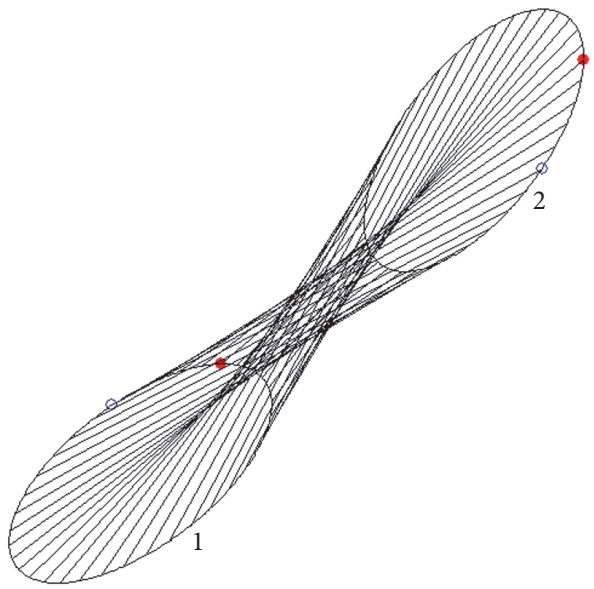

(a) Three-dimensional holographic spectra in $1 X$

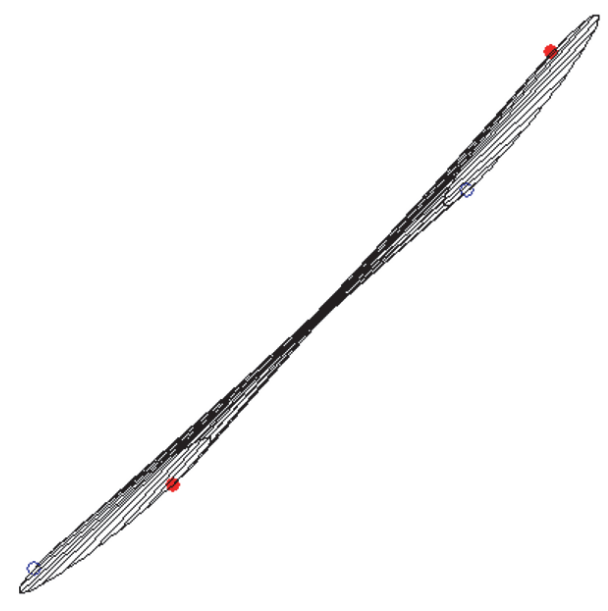

(c) Three-dimensional holographic spectra in $4 X$

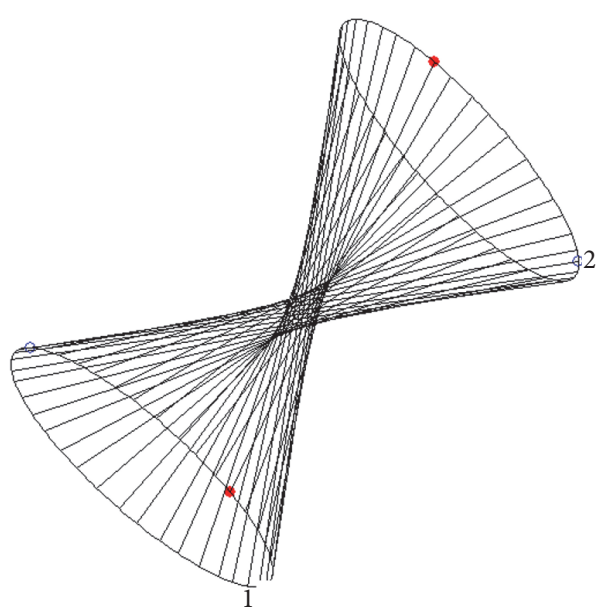

(b) Three-dimensional holographic spectra in $2 X$

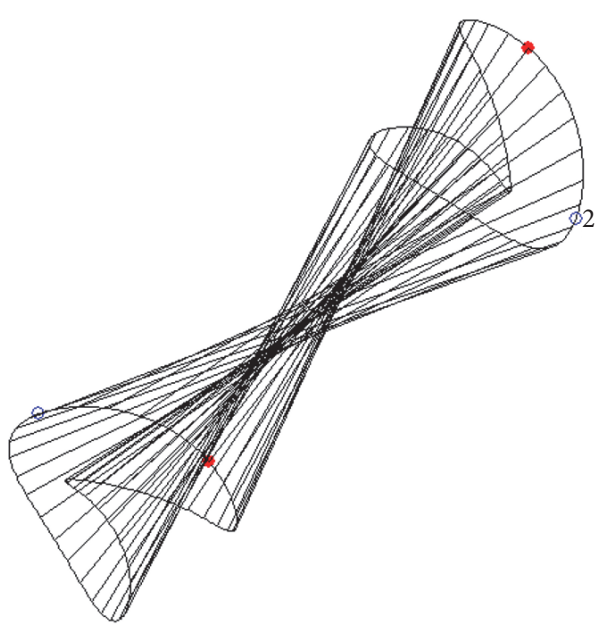

(d) Synthetic three-dimensional vibration in $1 X, 2 X$, and $4 X$

FIGURE 9: Three-dimensional vibration components.

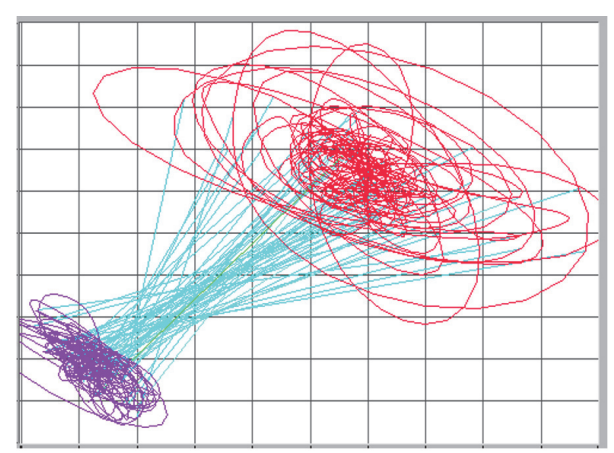

FIGURE 10: Filtered three-dimensional vibration with two sections projected in a two-dimensional plane.

fluid-induced vibration can be confirmed in this application. Moreover, if the conventional FFT is adopted, it is necessary to draw different cross section signal spectrums to observe the variations of the investigated signal. However, our method shows all rotor vibration information accompanied with the phase information using only an interface, which contributes to better identification of the different fault types.

\section{Conclusion}

To extract the vibration features of the compressor rotor, improve the fault identification ability, and thus promote prediction techniques, this paper proposes a three-dimensional spatial representation that includes three-dimensional vibration analysis and three-dimensional spatial orbit analysis. Using theoretical analysis and model construction and application, certain important advantages and comments are described as follows:

(1) The filtered and synthetic three-dimensional orbit analyses achieve accurate synthesis of different frequency components.

(2) The filtered three-dimensional orbit and the synthetic three-dimensional orbit extend the concept of the 
conventional orbit and produce total integration of the diagnostic information.

(3) The filtered three-dimensional orbit extracts the features of the vibration signal with a continuous band, and the synthetic three-dimensional orbit synthesizes certain frequency components according to the requirements.

(4) The filtered three-dimensional orbit enables a threedimensional orbit with noise to approach the true form, and the synthetic three-dimensional orbit can be integrated to display the selected frequency components with flexibility.

(5) The three-dimensional vibration analysis and the three-dimensional orbit analysis methods completely collect and rationally apply the fault information, which offers a new efficient and reasonable approach for fault diagnosis of a complex compressor and is beneficial for prediction.

\section{Competing Interests}

The authors declare that they have no competing interests.

\section{Acknowledgments}

This work was supported by the National Natural Science Foundation of China (nos. 51365051, 51405366, and 51421004) and the Program for New Century Excellent Talents in University (NCET-13-0461).

\section{References}

[1] K. YouFu, L. ShuLin, and L. YinHui, "Fauit diagnosis based on nonlinear complexity measure for reciprocating compressor," Journal of Mechanical Engineering, vol. 48, no. 3, pp. 102-107, 2012.

[2] L. S. Qu, Y. D. Chen, and X. Liu, "Discovering the holospectrum," Noise \& Vibration Control Worldwide, vol. 20, no. 2, pp. 58-62, 1989.

[3] L. Qu, X. Liu, G. Peyronne, and Y. Chen, "The holospectrum: a new method for rotor surveillance and diagnosis," Mechanical Systems and Signal Processing, vol. 3, no. 3, pp. 255-267, 1989.

[4] Y. H. Hu, L. Zhang, J. Z. Lin, L. F. Zhang, and M. R. Zhang, “The rotor system fault analysis based on diagnostic holographic spectrum," Journal of Vibration and Shock, vol. 12, no. 28, pp. 164-166, 2009.

[5] Y. Du and Z. Qin, "Holo-spectrum analysis of rotating machinery dynamic signals," Journal of Vibration, Measurement \& Diagnosis, vol. 22, no. 2, pp. 81-88, 2002.

[6] S. Liu and L. S. Qu, "Fault diagnosis prior the field balancing based on holospectrum," Journal of Vibration, Measurement and Diagnosis, vol. 24, no. 4, pp. 270-274, 2004.

[7] D. F. Shi and L. S. Qu, "Holospectrum decomposition as a diagnostic tool for rotating machinery," Journal of Xian Jiaotong University, vol. 32, no. 7, pp. 94-102, 1998.

[8] S. Liu and L. Qu, "Three-dimensional holospectrum in rotating machinery diagnosis," Journal of Xi'an Jiaotong University, vol. 38, no. 9, pp. 900-903, 2004.
[9] S. Liu, "A modified low-speed balancing method for flexible rotors based on holospectrum," Mechanical Systems and Signal Processing, vol. 21, no. 1, pp. 348-364, 2007.

[10] Y. J. Zhang, J. L. Zhang, Y. C. Zhang, and C. Dong, "Application of holospectrum technology on portable dynamic balancing instrument," Coal Mine Machinery, vol. 33, no. 3, pp. 201-203, 2012.

[11] Y. B. Ji, “The rotor balancing on either steady speed," Journal of Vibration Measurement \& Diagnosis, vol. 32, no. 4, pp. 596-600, 2012.

[12] S. Liu and L. Qu, "A new field balancing method of rotor systems based on holospectrum and genetic algorithm," Applied Soft Computing Journal, vol. 8, no. 1, pp. 446-455, 2008.

[13] G. R. Wen, X. N. Zhang, and M. Zhao, "Application of partical swarm optimization algorithm in field holo-balancing," in Lecture Notes in Computer Science, vol. 6328 of Lecture Notes in Computer Science, pp. 16-25, Springer, 2010.

[14] Y. G. Wei, B. P. Tang, F. B. Cheng, and X. F. Qin, "Research of holospectrum technique based on order tracking," Journal of Chongqing University (Natural Science Edition), vol. 30, no. 2, pp. 9-21, 2007.

[15] Y. C. Ni, Y. H. Liao, Z. C. Ma, and Q. C. Liu, "Research on calculation of order analysis and holospectrum of nonstationary signal methods for rotor," Compressor Blower \& Fan Technology, vol. 57, no. 4, pp. 19-26, 2015.

[16] H.-P. Wang, B.-P. Tang, Z.-Q. Su, Y. Han, and Y. Qin, “Order holospectrum analysis based on FT zoom correction," Journal of Vibration and Shock, vol. 33, no. 10, pp. 68-72, 2014.

[17] R. G. Wen, C. Jiang, Y. Li, and Y. Liao, "Fault feature extraction from the vibration signals in rotor start-up or slowdown processes based on order tracking and holospectrum," Journal of Vibration and Shock, vol. 35, no. 2, pp. 64-68, 2016.

[18] H. S. Li, S. Zhou, H. Zhu, and L. H. Zhao, "The method of holospectrumfault identification based on empirical mode decomposition," Journal of Vibration Measurement \& Diagnosis, vol. 31, no. 1, pp. 20-22, 2011.

[19] W. B. Zhang, X. Y. Zhou, and Y. Lin, "Harmonic window method and purified rubbing rotor axis orbit," Journal of Vibration Measurement \& Diagnosis, vol. 30, no. 1, pp. 87-90, 2010.

[20] F. Li, Y. Li, and Z. Wang, "Purification of axis orbit of rotor with generalized harmonic wavelet," Journal of Vibration, Measurement \& Diagnosis, vol. 28, no. 1, pp. 55-57, 2008.

[21] P. P. Sotiriadis, "Cascaded diophantine frequency synthesis," IEEE Transactions on Circuits and Systems I: Regular Papers, vol. 55, no. 3, pp. 741-751, 2008.

[22] Y. Qiu and G. Liu, "Frequency synthesis technology: history, actuality and development," Industrial Instrumentation \& Automation, vol. 5, pp. 12-14, 2005.

[23] P. Q. Cheng, Digital Signal Processing, Tsinghua University Press, Beijing, China, 2007.

[24] Y. Yang, J. Sun, X. Y. Zhao et al., "Experimental study of 3D trajectory of journal center of crankshaft bearings in multicylinder internal combustion engine," Journal of Mechanical Engineering, vol. 48, no. 3, pp. 174-179, 2012. 


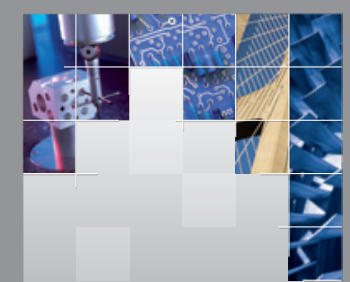

\section{Enfincering}
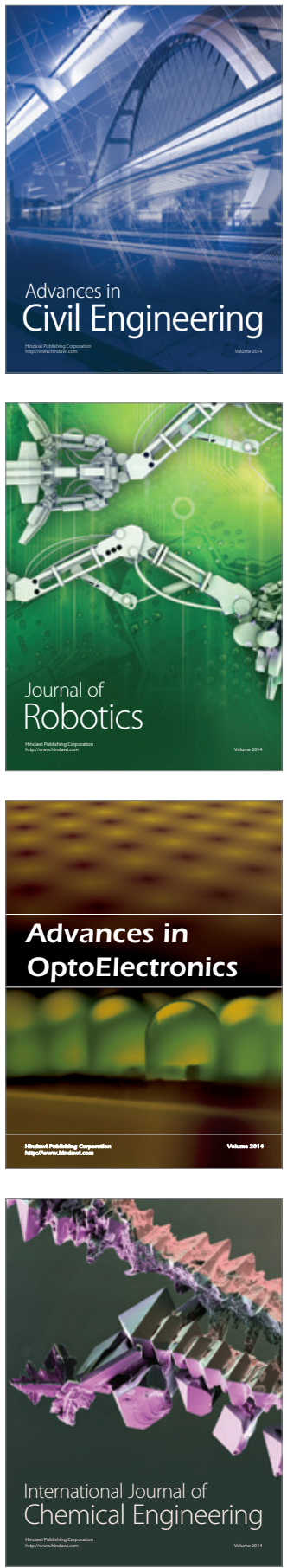

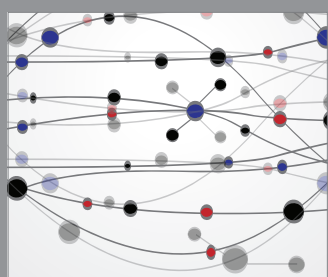

The Scientific World Journal

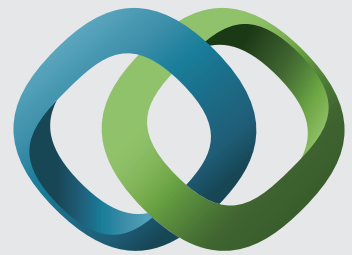

\section{Hindawi}

Submit your manuscripts at

http://www.hindawi.com
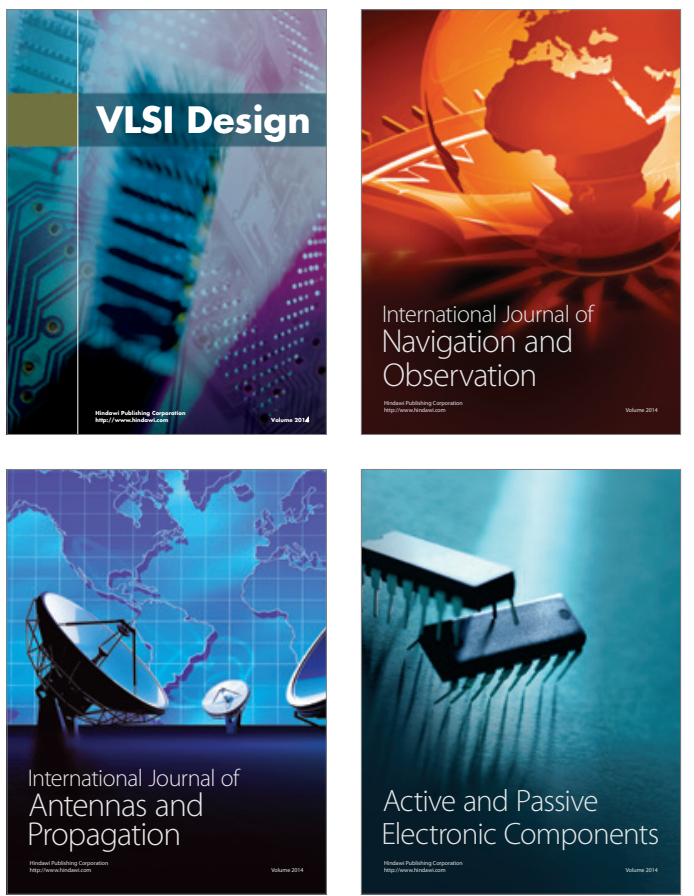
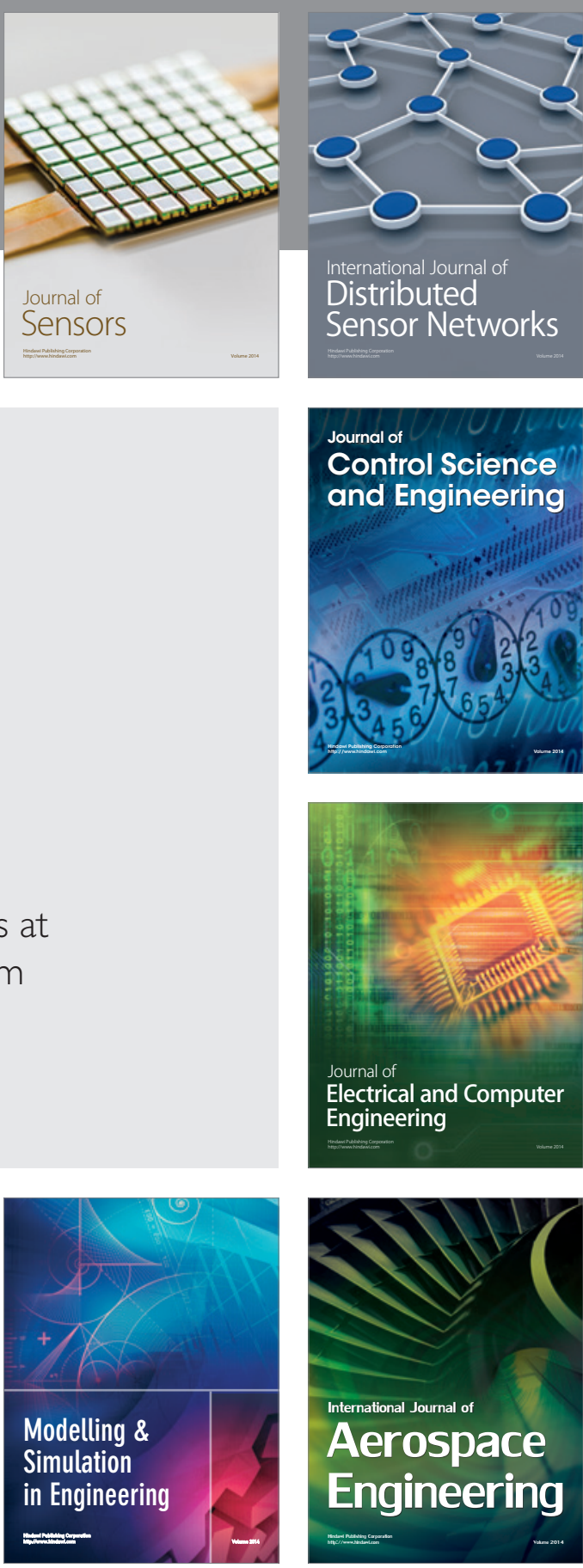

International Journal of

Distributed

Sensor Networks

Journal of

Control Science

and Engineering
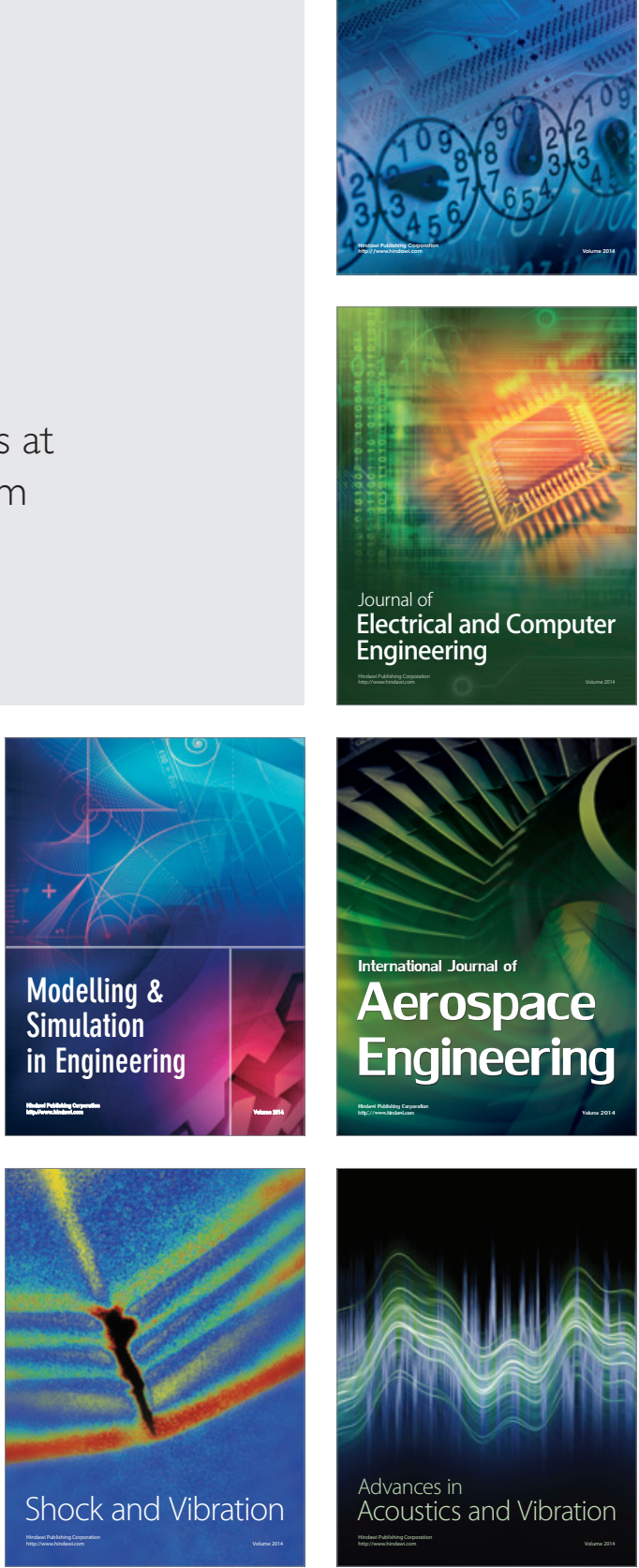